\title{
Leveraging service design by bridging business and process modeling
}

\author{
Francisco J. Pérez-Blanco, Juan Manuel Vara, Cristian Gómez, Valeria De Castro, Esperanza Marcos \\ Kybele Research Group, Universidad Rey Juan Carlos, Madrid, Spain \\ \{francisco.perez, juanmanuel.vara, cristian.gomez, valeria.decastro, esperanza.marcos\}@urjc.es
}

\begin{abstract}
There are currently several techniques or notations for business and process modeling that allow the idea of business to be explored in greater or less detail, while simultaneously helping to understand, conceptualize and represent the services that add value to an organization. These techniques have similarities and differences but are in many cases complementary. However, there is no solution that allows working with them in an integrated manner, shortening the distance between business and process modeling areas. All this given, this paper introduces the latest functionalities incorporated in a modeling environment for service design that currently supports 5 different notations (Business Model Canvas, $e^{3}$ value, Service Blueprint, Process Chain Network and BPMN) as well the partial generation of models from a model elaborated with a different notation along with the corresponding relations model.
\end{abstract}

\section{Introduction}

Literature reveals there is a huge number of definitions of what a business model is. Some academic ones are those provided by Teece [1], who argues that the definition of a business model implies identifying the way in which the company provides value to customers, attracts them so that they will pay for this value and converts that payment into profit, and Osterwalder and Pigneur [2], who argue that business models describe the bases upon which the firm creates, provides, and captures value.

The concept has been historically considered from three different perspectives: technology-oriented, strategy-oriented, and organization-oriented [3]. For instance, in the context of information technology, business models are almost immediately identified with process models, while in the context of organization theory, business models are conceived as an abstract representation of a company's structure or architecture.
Some authors even distinct four categories of business modeling, namely: business process models, business motivation models, business organization models and business rules models [2].

Two of these perspectives (business organization models and business process models) constitute the core of service design, which aims at helping in the development or improvement of services to deliver user-centered services by focusing on the interactions (or touchpoints) between the provider and the consumer [4]. Its main principles are human-orientation, value co-creation, process-based nature, tangible evidence, and holistic view. Born in the context of research on services marketing, service design evolved and gained impact [5] to finally establish as the entry point to service development for any organization seriously concerned about user experience, digital transformation, and the like [6]. See for instance the efforts of the British government in this line, materialized in the Government Digital Service initiative ${ }^{1}$.

Business modeling is indeed crucial to successful service design since in their approach towards a successful servitization process, companies need to redesign their business model constantly [7]. To that end it is essential that all the departments of the organization share a clear and common understanding vision of the business model, even when they speak different languages, which in the case of business models, implies using different notations [8]. This way, notations like the Business Model Canvas [9] or the $\mathrm{e}^{3}$ value model [10] serve to provide a quick and strategic overview of the organization (see Fig. 2). On the other hand, (business) process modeling techniques, like Service Blueprint [5], BPMN [11] or Process Chain Network (PCN) [12] are key to show the details of a particular service offering (see Fig. 3 and 4).

Even though tools supporting some of these techniques in an isolated manner exist, there is no comprehensive solution that makes it possible to work with several ones in an integrated way. There are

\footnotetext{
${ }^{1}$ https://gds.blog.gov.uk/
} 
also generic diagramming tools such as MS-Visio or Lucidchart, but while these solutions can be good options for quick sketching, they were not devised to enable the processing of the information collected in the models depicted with them.

This way, in order to fill the gap regarding proper tool support for modeling tasks in service design this paper addresses the following research questions:

RQ1: Which is the state of the art regarding tool support for modeling tasks in the context of service design?

RQ2: Is there a suitable set of modeling notations for service design?

RQ3: How can we provide tool support to use those notations in an integrated way?

As a result, this work introduces the latest improvements of INNoVaServ ${ }^{2}$., an open-source research project which provides a modelling toolkit that addresses the lack of tool support to bridge existing business and business process modelling notations for service design. To that end, it bundles a set of visual domain specific languages (DSL) plus the tooling needing to register and manage the relationships among the models elaborated with those DSLs in the context of a service design project. This way, apart from five visual DSLs (Business Model Canvas, $\mathrm{e}^{3}$ value, Service Blueprint, PCN, and BPMN) it provides a dashboard that supports the generation of partial models from models expressed with another notation, as well as a trace model that collects the relationships between both models. Besides, INNoVaServ supports the formal validation of Service Blueprint and PCN models [13][14] and more recently we have started to explore the integration of a DSL for Smart Contracts in order to support the generation of contracts from from $\mathrm{e}^{3}$ value models [15].

Since the core of this project is the development of a model-based tool that take the shape of a DSL toolkit, the guidelines sketched in [16] to build model-driven development frameworks have been followed.

To illustrate the use of the models and transformations supported by INNoVaServ you can refer to the artefacts developed in the context of a particular service design project used as case study. Its main objective was to tackle the issues that arose while managing a drastic growth (x2 in 6 years) in the number of students carrying out the defense of their respective final thesis projects at a public university. All the artifacts (models) developed in the project can be

\footnotetext{
${ }^{2} \mathrm{http}: / / \mathrm{www} . k y b e l e . e s /$ innovaserv/
}

accessed online ${ }^{3}$ and edited with the toolkit presented here.

Actually, some diagramming functionalities supported by INNoVaServ were briefly introduced in [17]. Note that being a tool-demo paper, the underlying methodological proposal was not covered at all. Consequently, the discussion regarding the role of modeling and this tool in the context of service design is completely new. Therefore, this work discuses which are the modeling notations better suited for each of the steps comprised in the double diamond process model (section 3). Furthermore, this paper focuses on the relationships between the modeling notations supported by the tool (section 4) and how these notations are integrated by means of technical bridges (section 5). Finally, we also discuss in a quantitative manner the results derived from using those technological bridges.

The rest of this paper is structured as follows: section 2 discusses related works; section 3 introduces the visual DSLs bundled in INNoVaServ by means of a case study; section 4 conceptually summarizes the correspondences identified among the different notations; section 5 presents the technical design of INNoVaServ and discusses some implementation details and section 6 concludes by highlighting the main contributions and providing directions for further work.

\section{Related works}

In the following, existing works in the area are reviewed from both the methodological and technical point of view in order to answer RQ1. It should be noted however that currently there is no work or tool supporting all the notations supported by INNoVaServ in an integrated manner.

A quick look at the plenty of systematic literature reviews on business process modelling and the topics covered by them shows that this is somehow the most mature business modelling discipline. Recent reviews are indeed not focused on characterizing existing proposals, since that has been largely done in the past, but on available mechanisms to assess their quality [18] or complexity [19].

However, despite the number of works in the area, still new approaches for business process modelling [20] and BPMN dialects [21] appear every so often. Many of them are focused indeed on shortening the distance between professionals from business areas and business process modelling notations [22].

By contrast, instead of defining yet-another business process modelling language tailored to business professionals, the aim of INNoVaServ is at providing

\footnotetext{
${ }^{3}$ http://kybele.es/innovaserv/finalthesis/
} 
them with tool support for the languages they are already using. At the same time, providing support to strategy and organization - oriented business modelling notations like Business Model Canvas or $\mathrm{e}^{3}$ value, will help to shorten the historical distance between IT and business practitioners. The models defined and handled by management areas become directly connected (or even mapped) to the models used by IT practitioners, more frequently expressed in terms of BPMN or UML.

On the other hand, despite the recent interest attracted by the field due to the rise of product-service-systems [6], business intelligence modelling [23] and some other disciplines, research on strategic- and organization-oriented business modelling, is still at an early stage, probably because the business process model hype preceded the business model one [8].

Regarding tool-support, provided that no tool has been found supporting the five notations integrated in INNoVaServ and exploiting the relationships between them, some of the existing tools supporting at least two of them are briefly discussed in the following.

First of all, there are some academic works, like the one from Efendioglu \& Woitsch [24], which supports the Business Model Canvas and $\mathrm{e}^{3}$ value, or the one from Ateetanan et al. [25], which combines business architecture models, Service Blueprint and BPMN. These are consequently model-based solutions for business or business process modeling like INNoVaServ. However, they are focused just on one of these areas so they are not able to bridge both worlds such as INNoVaServ does.

There exist also some non-academic tools supporting some of these notations, like Canvanaizer, Miro, draw.io, Lucidchart or Gliffy, which are web-based applications that supports (some of them) collaborative edition of Business Model Canvas, Service Blueprint or BPMN. They have simple and intuitive graphical interfaces, but they are not model-based tools, so subsequent processing of the information collected in those models is not contemplated. Likewise, they lack exporting capabilities supporting suitable formats for post-processing (like XML). Finally, some of them are commercial solutions offering free limited editions, while others are completely free.

Likewise, tool support for $\mathrm{e}^{3}$ value was so far limited to the e3editor, a desktop application that allows representing graphically and accurately $\mathrm{e}^{3}$ value diagrams. Models can be persisted in RDF format, which simplifies export/import tasks. Regarding PCN, no tool has been found supporting this notation. The only way of defining PCN diagrams to date was again using generic diagramming apps or even image editors, like MS Visio or Lucidchart.

Finally, as already mentioned, there are plenty of BPMN tools, such as Bonita Studio, Signavio, BizAgi or IBM WebSphere, each one providing different capabilities.

All this given, to the best of our knowledge this is the first proposal to consider the business and process modelling notations discussed here along with the exploit of its correspondences and providing tool support to use them in the context of service design.

\section{Business and process modeling with INNoVaServ}

In order to answer RQ2, we first needed to choose a kind of standard process model for Service Design to later discuss the role of modeling in such process model. To that end, we focus on the Double Diamond model from the UK Design Council since it is widely acknowledged as one of the most adopted process models for Service Design, even though there might be some other options like those from IDEO or the Hasso-Plattner Institute. [26].

The model defines a design process encompassing two different phases of convergent and divergent thinking. During the first one, given that the aim is at understanding the problem in a global way (discovering the user needs to later define the problem that will be tackled), business modeling can help. Later on, the second phase aims to design the solution (design multiple solutions to the problem identified and to go deep into the details of the solution selected which will be finally delivered), so business process modeling is frequently used during this stage. All this given, Fig. 1 illustrates the methodological and technical proposal of INNoVaServ by sketching how the specific set of notations currently supported by the toolkit fit into the Double Diamond service design process.

Given that the first stage of the process requires a global vision of the context, two notations that provides different global points of view were selected: Business Model Canvas and $\mathrm{e}^{3}$ value. The second stage, however, requires from models that allow designing a solution, which inherently comprises a process. Notations which provides different points of view of a given process were thus selected. Note that while Service Blueprint offers a simplified vision of the process, BPMN allows for much more detail, while PCN allow dealing with aspects that BPMN cannot cover such as monetary or non-monetary profits or losses.

Taking for granted that there are other business and process modeling notations that could be selected for each stage, the election was mainly based on the 


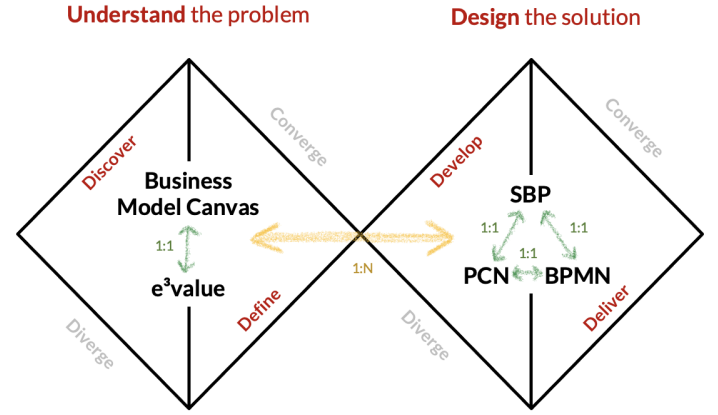

Figure 1. INNoVaServ models in the context of the double-diamond service design process.

popularity of these notations and, in some case, like that of PCN, on the potential of this notation to cover aspects not covered by other languages. Note also that the aim is at providing a set of notations so that each designer could choose those that fit better with their expertise, preferences or needs. In the following we elaborate a bit on the use of the models specifically supported by INNoVaServ in the context of the double diamond model.

\subsection{Understanding the problem}

Business Model Canvas and the $\mathrm{e}^{3}$ value are useful at the early stages of the classical double-diamond service design process because they help to understand the business context, which is the main goal of the Exploration and Creation steps.

The analysis of the data collected in these models, along with the underlying information, was used during the creation stage to generate an action plan that can articulate the results of the exploration phase. In this case, the main conclusion was that the key issues in the final thesis projects area were related with the assignment of final thesis to tutors or supervisors and students. Being this a manually operated service, there was a need to automatize or optimize the way this service is provided in order to accelerate the process.

As regards Business Model Canvas, it is perhaps the most popular business model, especially among business people. It helps to visualize and evaluate a specific value proposition which combines products and services offering and is built around nine basic modules which contain certain information to represent a company's logic to earn money [9].

Fig. 2 shows the Business Model Canvas depicted to describe the different assets and stakeholders related with the main actor of this scenario: the Unit of Undergraduate Thesis. Among other information, we can see that the key partners of this Unit are the supervisors and two software providers supporting the necessary systems.

The Business Model Canvas provides a global vision of the organization, without going deep into value exchanges or service operations, something that is slightly possible with the next notation that we are going to see: $\mathrm{e}^{3}$ value. A major disadvantage of the Business Model Canvas is that it does not support the visualization of the elements related to the different value propositions (goods or services) in which the organization is involved, since the model by nature is devised to support the analysis of one value proposition at a time. By contrast, being able to see all of an organization's production activities at once is especially useful when designing product-service offerings [6].

The $\mathrm{e}^{3}$ value is a business model which allows the graphical representation of a business idea, without going into the details of the processes composing the services offered. To that end, $\mathrm{e}^{3}$ value models are focused on value activities, which are the activities performed by an actor to obtain a certain benefit, and value exchanges, where actors (unitary or market segments) exchange different value objects (goods, money or even services) [10].

Regarding the $\mathrm{e}^{3}$ value diagram for the management of undergraduate thesis projects (Fig. 2), note that it contains some elements that match with other elements of the Business Model Canvas, like the actors and some value objects.

\subsection{Developing the solution}

After having identified the relevant issues exploring the problem space, we shall provide solutions to such problems. In order to do so, INNoVaServ supports 3 business process modeling notations to define the service operations that should serve to address the problems identified: Service Blueprint, PCN and BPMN.

The Service Blueprint is a graphical tool for the design of business service operations, which is focused on detailing the interaction between the customer and the service provider in the provision of a given service [27]. This way, since every Canvas or $\mathrm{e}^{3}$ value model entails typically several service operations, a Service Blueprint is needed to visualize the details of each service operation.

For instance, the Service Blueprint model shown in Fig. 3 was defined to design a service that helped on the management of the final thesis life cycle, along with a supporting application. Note that it gathers all the interactions between the student (service consumer) and its supervisor (service provider), as well as the internal 

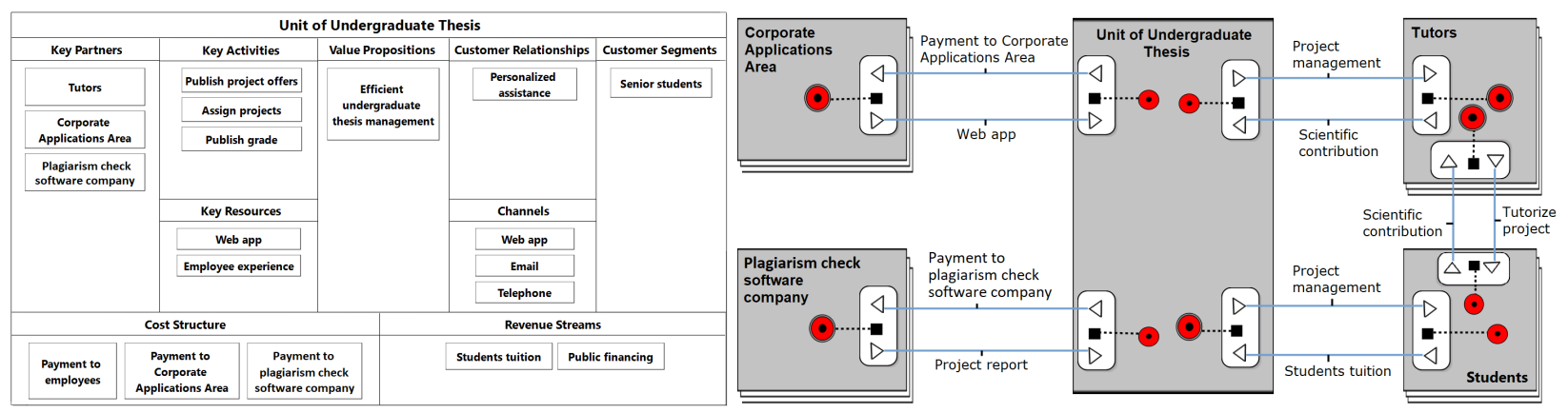

Figure 2. Left: Business Model Canvas made with INNoVaServ. Right: $\mathrm{e}^{3}$ value model made with INNoVaServ.

and supporting processes.

However, decision nodes cannot be represented in Service Blueprints, which can interfere with the visualization of processes based on contextual conditions. Besides, it only supports the representation of two entities (service consumer and provider).

On the other hand, the PCN, another service modeling technique to visualize service operations proposed by Scott E. Sampson [12], offers more detail than Service Blueprint, which does not differentiate customer independent actions nor reflect the other participant entities. PCN is able to do so due to its networked nature. This way, in spite of being much less popular than Service Blueprints, probably due to a higher complexity and the lack of tool support (until the advent of INNoVaServ), PCN can complement Service Blueprint to enable the comprehensive modeling of service operations

Fig. 3 shows the PCN diagram that was elaborated to present the new management process to the administrative staff of the university. Note that 3 regions are distinguished in every entity. Collateral regions correspond to the areas of direct interaction (Dir.), where one to one relationships are represented. Next, the areas of surrogate interaction (Sur.) contain the steps of the process that involve some action of the entity on the material resources of another entity. Finally, the independent process region (Ind.) is where the tasks performed by the entity without external interactions are represented. Along with tags to reflect where monetary or non-monetary profit and loss are produced, it is a differentiating characteristic from other process models as BPMN.

Finally, to bring the previous models closer to IT practitioners, a graphical DSL to allow sketching process operations with BPMN [11] has been also bundled in INNoVaServ. Fig. 4 shows the BPMN diagram generated from the previous Service Blueprint for the case study. This model was the preferred one by the IT staff when they addressed the development of the web application that should definitively alleviate the problem tackled in the project.

All in all, the models defined with these notations play a key role during the reflection and implementation stages of the service design process, since they help to develop prototypes and test solutions. During the reflection stage, Service Blueprint and (or) PCN models are frequently used by business people to discuss the details of the service operations that must be implemented in order to operate the services offering identified throughout the exploration and creation stages. Afterwards, having taken into account that IT practitioners are more familiar with BPMN, the Service Blueprint and PCN models are used as input to automatically generate the BPMN models that will drive the implementation.

Key message is that the notations supported by INNoVaServ are somehow complementary and any of them can be used at any given moment depending on different factors, such as the expertise of the organization or the level of detail with which service operations need to be represented at that particular moment. In this context, the ability of identifying and registering the relationships between business models expressed with these notations is key to enable a clear and common understanding that eases strategic decision making and the later implementation of those decisions. As a first step in this direction, next section analyzes the conceptual correspondences among the different notations supported by INNoVaServ and how we have exploited them.

\section{Correspondences analysis}

So far, we have shown that the models currently supported by INNoVaServ can be used when designing the services provided by an organization. To answer RQ3 and find a way to use them in an integrated way, we have analyzed the relationships between the notations or modeling languages currently supported by the tool. 


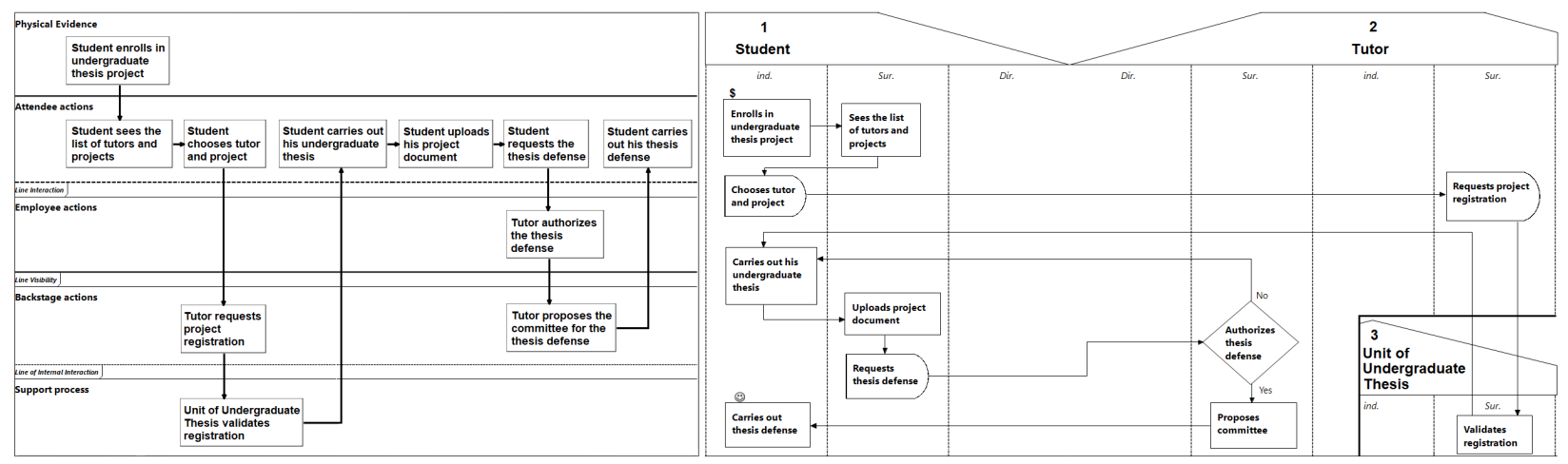

Figure 3. Left: Service Blueprint model made with INNoVaServ. Right: PCN model made with INNoVaServ.

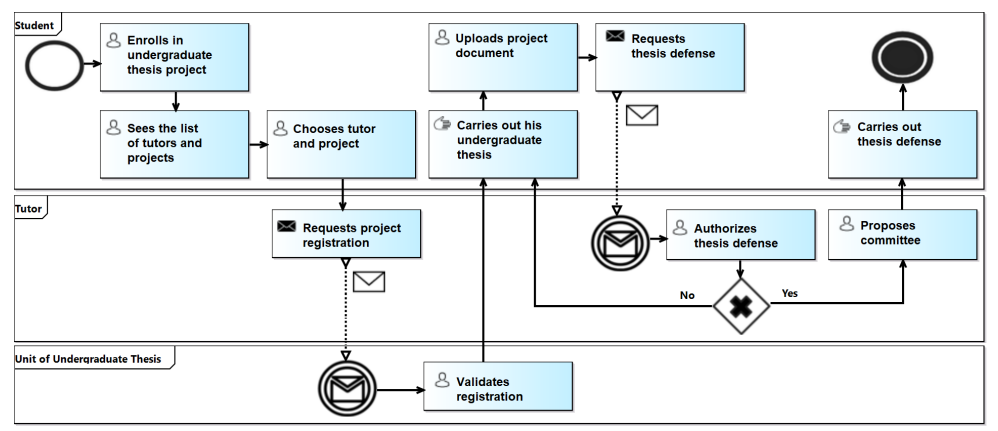

Figure 4. BPMN model generated with INNoVaServ from a PCN model.

Due to the different nature of the notations (what can be appreciated in Fig.1), this study has been divided into three parts: relations between business models (Canvas and $\mathrm{e}^{3}$ value); relations between process modeling notations (Service Blueprint, PCN and BPMN); relations between the two previous groups.

Regarding the first part of the analysis, Fig. 5 shows an excerpt of an hypothetical model of conceptual relationships between the Canvas and the $\mathrm{e}^{3}$ value models. On the left-hand side of the figure the main concepts of Business Model Canvas are shown, whereas those of $\mathrm{e}^{3}$ value are shown on the right-hand side.

After studying different case studies, it has been concluded that the key partners of a Business Model Canvas, mostly correspond to the actors of an $\mathrm{e}^{3}$ value model (see Fig. 2 for instance). In addition, all the values contained in the different sections of a Business Model Canvas, apart from the customer segments and key partners, match with the value objects of an $\mathrm{e}^{3}$ value model.

On the other hand, Fig. 6 summarizes the conceptual relationships identified between PCN, BPMN and Service Blueprint. Note that the number of similarities found identified is much higher as it is the number of modeling elements comprising these notations.

For instance, entities in a PCN model can be

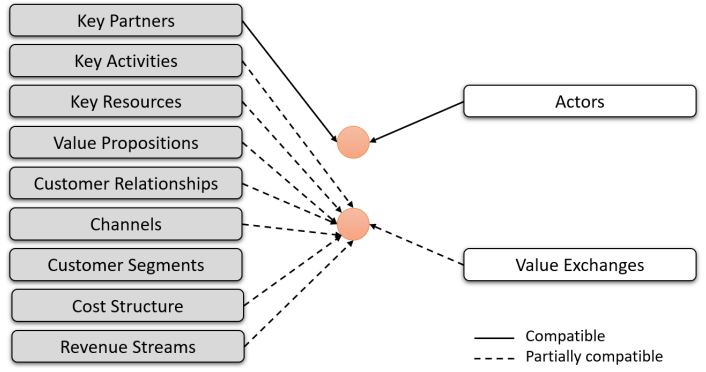

Figure 5. Correspondences between Business Model Canvas and $\mathrm{e}^{3}$ value.

directly translated into BPMN lanes. However, this is not possible in the case of Service Blueprint since only two different entities can be represented in any given Service Blueprint: the customer and the provider. More complex relationships are those that take place between the wide variety of actions and dependencies available in both PCN and BPMN (standard steps, waiting steps, decision nodes, standard dependencies, delayed dependencies, etc.). The case is much simpler with Service Blueprint since it only considers one type of actions and dependencies.

Finally, the third step of this analysis is to analyze the relationships between both types of models (business 


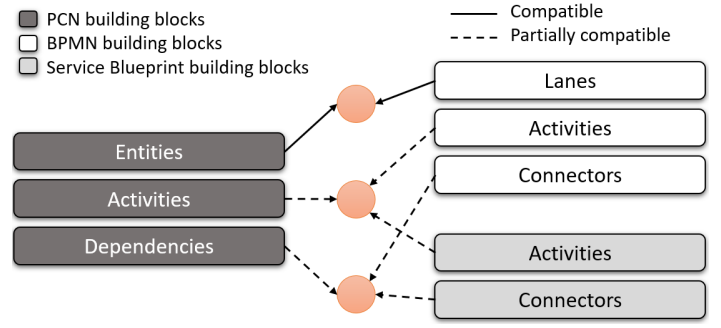

Figure 6. Correspondences between PCN, BPMN and Service Blueprint.

and process models). Given that the first kind of models focuses on representing the structure of an organization, while the latter focus on the description of the service operations, the main relationship can be summarized as follows: process models are used to expand the information collected in business models. Likewise, from the data contained in a Canvas model (such as key activities) or in an $\mathrm{e}^{3}$ value model (such as value exchanges), the number of process models needed to represent the main service operations can be estimated. For example, since the $\mathrm{e}^{3}$ value diagram of this case study (see Fig. 2) comprises three value exchanges, at least three Service Blueprint models will be needed to represent the processes that enable such value exchanges.

Since we are considering five different notations, we can bridge them with twenty model transformations. To address this complexity, we have selected the minimum set of transformations that allow generating models for any supported notation by means of transformation chains. This way, the direct transformations implemented and bundled in INNoVaServ are those shown in Fig. 7.

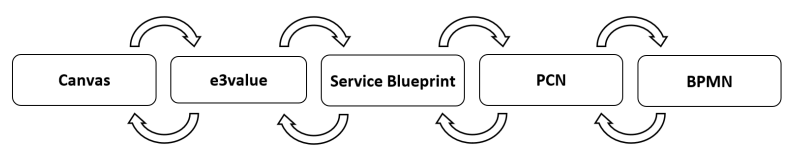

Figure 7. Direct transformations bundled in INNoVaServ.

After implementing the correspondences found by means of model transformations (see section 5), the completeness of these transformations in terms of the number of model elements that they can map was analyzed. Therefore, the analysis consists basically of identifying the number of model elements generated by each transformation, regarding the number of elements comprising the model in case it was developed by hand from scratch. We did so using 5 different case studies, namely: Deliveroo, a food delivery service; Car2go, a world-wide carsharing service; BiciMAD, the Madrid public bike rental service; an online betting house and a neuropsychology and psych pedagogy institute. For each of these case studies, the corresponding set of business and process models were developed from scratch using INNoVaServ.

Next, the transformations bundled in INNoVaServ were run using the models manually hand-crafted as source models (considered as complete models) and the models generated were manually compared with the correspondent handmade model in order to compute the percentage of model elements generated by each transformation. In this sense, each element is essentially an object resulting from instantiating the corresponding metamodel. For instance, in the case of Service Blueprint (see Fig. 3), the board, action boxes, action texts and connections are the elements considered.

All this given, Table 1 shows the percentages of model elements generated on average by each transformation for the five case studies considered. The data gathered support the initial and logical intuition: It should be highlighted that the mappings between models in the same category (either business or business process models) are more comprehensive than those between models of different categories. This is due to the different nature of business and process models. For instance, $75,59 \%$ of the $\mathrm{e}^{3}$ value model can be generated from the related Business Model Canvas, whereas $67,46 \%$ of the BPMN model can be generated from the related PCN model. On the contrary, only $7,75 \%$ of the Service Blueprint can be generated from the corresponding $\mathrm{e}^{3}$ value model.

Table 1. Percentage of model elements generated by INNoVaServ

\begin{tabular}{l|r}
\hline Transformation & \% AVG \\
\hline Canvas from $\mathrm{e}^{3}$ value & $36.76 \%$ \\
$\mathrm{e}^{3}$ value from Canvas & $75.59 \%$ \\
$\mathrm{e}^{3}$ value from Service Blueprint & $3.23 \%$ \\
Service Blueprint from $\mathrm{e}^{3}$ value & $7.75 \%$ \\
Service Blueprint from PCN & $97.71 \%$ \\
PCN from Service Blueprint & $44.98 \%$ \\
PCN from BPMN & $89.65 \%$ \\
BPMN from PCN & $67.46 \%$ \\
\hline
\end{tabular}

Note however, that such information is really relevant since it eases the transition from high-level service design, where the service offering or the organization is conceived and discussed, to low-level service design, where that offering gives rise to a number of service operations that will be designed by means of process models. The information gathered from the business model serves to provide rough versions of those process models to be completed by the 
service design team.

From the data gathered, we can conclude that the partial models generated by the tool are a very good starting point. In four of the eight scenarios considered, more than $67 \%$ of the target model is generated, whereas that percentage ranges between $36 \%$ and $50 \%$ for another couple of scenarios. Note also that the two scenarios below the $8 \%$ in terms of model elements generated correspond to the transformation from business to process models, something logical since process models own a deeper level of detail.

Note that the automation of these transformations is still one of the most interesting contributions in the context of the service design process, since they will guide the transition from decision making to service development in the form of service operations design. If we make an analogy with the more traditional conception of the software development process, we could say that these transformations guide the way down from the analysis to the design phase.

\section{Technological solution}

The conceptual architecture and some technical details of the modeling toolkit build to support the methodological proposal of this work were briefly discussed in [17] as part of a demo. Fig. 8 summarizes such conceptual architecture and this section focuses on describing the new features integrated in the toolkit.

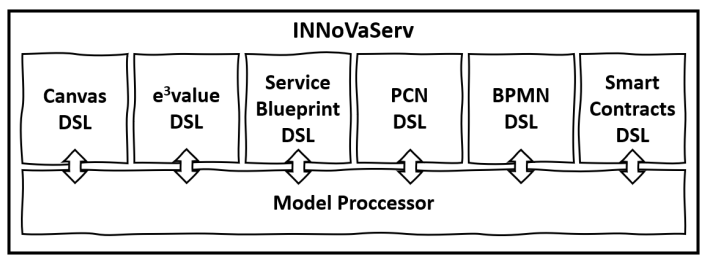

Figure 8. Simplified overview of INNoVaServ's conceptual architecture.

INNoVaServ was built to address the lack of tooling supporting business and process modeling in an integrated manner and to allow the post-processing of the information collected [16]. To that end, INNoVaServ integrates a set of visual DSLs developed with Eclipse EMF/GMF and Sirius to support five different notations, namely the Business Model Canvas, the $\mathrm{e}^{3}$ value, the Service Blueprint, the PCN and the BPMN. In recent works we have also explored the possibility of integrating a DSL for Smart Contracts development to bring the use of smart contracts to service design [15]. These DSLs are completely independent in the sense that they have different data models but are connected by means of a model processor (to allow transformations and validations). This fact provides modularization and eases the development process.

As well, current version of INNoVaServ bundles the transformations discussed in section 4 . These transformations also generate a trace model collecting the correspondences between the models involved in the transformation. Fig. 9 shows an excerpt from the traces model generated when moving from the Business Model Canvas to the $\mathrm{e}^{3}$ value of the case study. Furthermore, the tool supports other functionalities like automatic validation and correction of models.

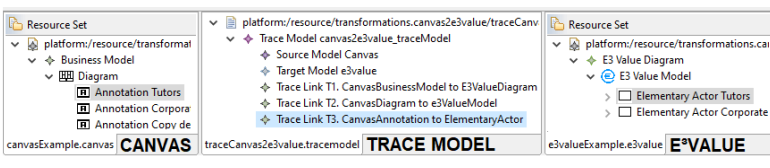

Figure 9. Trace model between a Business Model Canvas and $\mathrm{e}^{3}$ value.

Given that a certain number of potential user roles fit in the area of business management, we felt that an effort in terms of improving the tool's usability was needed, albeit we are dealing with a research prototype. To that end, a graphical dashboard providing an intuitive and agile manner to manage the different models used during the service design process has been implemented (Fig. 10). This panel provides control for the creation, selection, deletion and transformation of models.

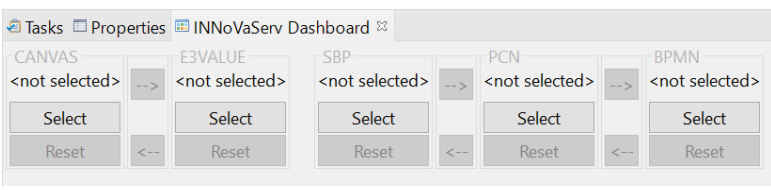

Figure 10. First version of the INNoVaServ dashboard.

As can be seen in Fig. 10, the dashboard is divided into two large blocks to locate respectively the business and process models of the project. Once a model is selected, the controls that allow to use it as source model to run any of the bundled transformations are enabled, substantially improving the usability of the toolkit regarding previous versions of the tool.

We are now working to improve the transformation from business to process models. For instance, every $\mathrm{e}^{3}$ value model yields as many process models as value exchanges are represented. A way to collect these relationships in the dashboard would be to replicate the block of process models for each value exchange comprised in the value model. 


\section{Conclusion and future work}

This work has presented INNoVaServ, a modeling toolkit for service design that provides an integrated environment which bundles five DSLs to support the elaboration of Business Model Canvas, $\mathrm{e}^{3}$ value, Service Blueprint, PCN and BPMN models.

Regarding RQ1, section 1 and section 2 have analyzed the role of modeling and modeling tools in service design. Next, in order to answer RQ2, section 3 has identified a set of modeling notations for service design, showing how they fit in the double diamond design process model. Finally, section 4 have analyzed the relationships between such notations in response to RQ3, showing how they are exploded by means of technological bridges based on model processing techniques [16]. These bridges allow to capitalize that knowledge and enable the automatic identification of the relationships that hold between models expressed with different notations. To that end, the toolkit supports the generation of partial views of a model from another model expressed with a different notation and the gathering of those relationships in relational models [28]. Likewise, we have included into INNoVaServ a dashboard to support in an integrated and intuitive manner the management of the models based on different notations used during any given service design project.

Regarding RQ2, we would like to stress that the selection of notations is not the result of a scientific process but of our own experience and personal reflections. A completely different selection could be made and this is actually one of the strengths of the proposal: the tool is very extensible so that other notations can be easily added to the set of notations currently supported by the tool. We just need to implement a new graphical DSL and a set of technological bridges to connect the newly added notation with those already bundled in the tool. We have the experience and know-how to do so in a straight forward manner with limited time and resources.

Regarding further work, we are now working in the validation of the proposal by conducting an empirical study of some depth, although we have already started to show that it can be useful, for instance, to integrate the use of smart contracts in the electronic administration [15].

Probably the most interesting future work we have addressed is the connection between the process models used during the service design project with the data generated by the daily operations or the organization under study. Enriching these models with high level information generated from these data will facilitate the identification of strengths and potential weaknesses and could guide or facilitate strategic decision making in the organization. To that end, we are enriching existing process mining tools so that INNoVaServ models can be directly used in such tools.

All in all, INNoVaServ addresses an issue that has been acknowledged as one of the main problems of service design: the lack of proper technical support [6]. The constant and rapid development of new services, goods or product-service offerings to address emerging needs as soon as they appear is indeed a must for any organization. Hence, the increasing interest regarding the field of service design. However, service design is currently considered an emerging field, in which industry is probably ahead of academia. Bringing some degree of formalization to the field should contribute to ensure the success of the process and to increases the effectiveness of the artefacts delivered, thus improving the rationality of the decisions within the company.

Without harming the creativity of designers while still fostering co-creation, model-based processing techniques make it almost immediate to start asking questions to the design artifacts. For instance, the organization is now able to: analyze the $\mathrm{e}^{3}$ value model in order to assess if it is involved in too many value exchanges and give back such assessment with quantitative data; compare two Business Model Canvas to identify the number of new partners that appeared; apply process mining techniques in order to assess the performance of a particular process and compare it with the process modeled in a Service Blueprint or a BPMN model; use formal techniques to validate or simulate the service operations implied in the provision of a given service, etc. A wide range of possibilities appears as soon as designers are able to persist and post-process their models instead of using them as mere sketching tools.

Finally, this work is somehow a step forward in the line we started some time ago, in which our objective is not so much to innovate in the area of model-driven engineering per se as to show the utility of model-based technologies in other areas. In this sense, we are aligned with the already expressed thought that MDE has achieved certain levels of maturity but needs from a more applied or realistic point of view [29]. One movement in this direction is to broaden its scope of application to other fields where both modelling and automation can decisively help to address their problems of interest. This is the case with the people from business management areas: model-driven engineering practitioners can help them to materialize and drive forward their ideas by providing them with the appropriate model-based tooling. 
Acknowledgements. This work has been partially funded by the Regional Government of Madrid, through the FORTE-CM project (S2018/TCS-4314) and the Spanish MINECO, through the MADRID project (TIN2017-88557-R).

\section{References}

[1] D. J. Teece, "Business models, business strategy and innovation," Long range planning, vol. 43, no. 2-3, pp. 172-194, 2010.

[2] D. M. Bridgeland and R. Zahavi, Business modeling: a practical guide to realizing business value. Morgan Kaufmann, 2008.

[3] B. W. Wirtz, A. Pistoia, S. Ullrich, and V. Göttel, "Business models: Origin, development and future research perspectives," Long range planning, vol. 49, no. 1, pp. 36-54, 2016.

[4] L. S. Cook, D. E. Bowen, R. B. Chase, S. Dasu, D. M. Stewart, and D. A. Tansik, "Human issues in service design," Journal of operations management, vol. 20, no. 2, pp. 159-174, 2002.

[5] L. Shostack, "Desing services that delivery," Harvard Business review, vol. 84115, 1984.

[6] S. Cavalieri and G. Pezzotta, "Product-service systems engineering: State of the art and research challenges," Computers in industry, vol. 63, no. 4, pp. 278-288, 2012.

[7] S. Holmlid and S. Evenson, "Bringing service design to service sciences, management and engineering," in Service science, management and engineering education for the 21st century, pp. 341-345, Springer, 2008.

[8] C. M. DaSilva and P. Trkman, "Business model: What it is and what it is not," Long range planning, vol. 47, no. 6, pp. 379-389, 2014.

[9] A. Osterwalder and Y. Pigneur, Business model generation: a handbook for visionaries, game changers, and challengers. John Wiley \& Sons, 2010.

[10] J. Gordijn, "E-business value modelling using the e3-value ontology," in Value creation from e-business models, pp. 98-127, Elsevier, 2004.

[11] M. Chinosi and A. Trombetta, "Bpmn: An introduction to the standard," Computer Standards \& Interfaces, vol. 34, no. 1, pp. 124-134, 2012.

[12] S. E. Sampson, "Visualizing service operations," Journal of Service Research, vol. 15, no. 2, pp. 182-198, 2012.

[13] M. Estañol, E. Marcos, X. Oriol, F. J. Pérez, E. Teniente, and J. M. Vara, "Validation of service blueprint models by means of formal simulation techniques," in International Conference on Service-Oriented Computing, pp. 80-95, Springer, 2017.

[14] E. Gómez-Martínez, F. Pérez-Blanco, J. de Lara, J. M. Vara, and E. Marcos, "Formal support of process chain networks using model-driven engineering and petri nets," in Proceedings of the 34th ACM/SIGAPP Symposium on Applied Computing, pp. 98-100, 2019.

[15] C. Gómez, F. J. Pérez Blanco, J. M. Vara, V. De Castro, and E. Marcos, "Design and development of smart contracts for e-government through value and business process modeling," in Proceedings of the 54th Hawaii International Conference on System Sciences, p. 2069. 2021.
[16] J. M. Vara and E. Marcos, "A framework for model-driven development of information systems: Technical decisions and lessons learned," Journal of Systems and Software, vol. 85, no. 10, pp. 2368-2384, 2012.

[17] F. J. Pérez-Blanco, J. M. Vara, C. Gómez, V. De Castro, and E. Marcos, "Model-based tool support for service design," in International Conference on Fundamental Approaches to Software Engineering, pp. 266-272, Springer, 2020.

[18] I. M.-M. de Oca, M. Snoeck, H. A. Reijers, and A. Rodríguez-Morffi, "A systematic literature review of studies on business process modeling quality," Information and Software Technology, vol. 58, pp. 187-205, 2015.

[19] G. Polančič and B. Cegnar, "Complexity metrics for process models-a systematic literature review," Computer Standards \& Interfaces, vol. 51, pp. 104-117, 2017.

[20] M. Estanol, A. Queralt, M. R. Sancho, and E. Teniente, "Artifact-centric business process models in uml," in International Conference on Business Process Management, pp. 292-303, Springer, 2012.

[21] J. Solís-Martínez, J. P. Espada, B. C. P. G-Bustelo, and J. M. C. Lovelle, "Bpmn musim: Approach to improve the domain expert's efficiency in business processes modeling for the generation of specific software applications," Expert Systems with Applications, vol. 41, no. 4, pp. 1864-1874, 2014.

[22] E. Umuhoza, M. Brambilla, D. Ripamonti, and J. Cabot, "An empirical study on simplification of business process modeling languages," in Proceedings of the 2015 ACM SIGPLAN International Conference on Software Language Engineering, pp. 13-24, 2015.

[23] J. Horkoff, D. Barone, L. Jiang, E. Yu, D. Amyot, A. Borgida, and J. Mylopoulos, "Strategic business modeling: representation and reasoning," Software \& Systems Modeling, vol. 13, no. 3, pp. 1015-1041, 2014.

[24] N. Efendioglu and R. Woitsch, "A modelling method for digital service design and intellectual property management towards industry 4.0: Caxman case," in International Conference on Serviceology, pp. 153-163, Springer, 2017.

[25] P. Ateetanan, S. Usanavasin, K. Shirahada, and T. Supnithi, "From service design to enterprise architecture: The alignment of service blueprint and business architecture with business process model and notation," in International Conference on Serviceology, pp. 202-214, Springer, 2017.

[26] K. Tschimmel, "Design thinking as an effective toolkit for innovation," in ISPIM Conference Proceedings, p. 1, The International Society for Professional Innovation Management (ISPIM), 2012.

[27] M. J. Bitner, A. L. Ostrom, and F. N. Morgan, "Service blueprinting: a practical technique for service innovation," California management review, vol. 50, no. 3, pp. 66-94, 2008.

[28] J. M. Vara, V. A. Bollati, Á. Jiménez, and E. Marcos, "Dealing with traceability in the mddof model transformations," IEEE Transactions on Software Engineering, vol. 40, no. 6, pp. 555-583, 2014.

[29] J. Whittle, J. Hutchinson, and M. Rouncefield, "The state of practice in model-driven engineering," IEEE software, vol. 31, no. 3, pp. 79-85, 2013. 